Revue d'histoire de l'Amérique française

ZRS REVUE D.HISTOIRE DE L'AMÉRIQUE FRANÇAISE

\title{
Remarques sur la notion de culture en anthropologie
}

\section{Marcel Rioux}

Volume 4, numéro 3, décembre 1950

URI : https://id.erudit.org/iderudit/801651ar

DOI : https://doi.org/10.7202/801651ar

Aller au sommaire du numéro

Éditeur(s)

Institut d'histoire de l'Amérique française

ISSN

0035-2357 (imprimé)

1492-1383 (numérique)

Découvrir la revue

Citer cet article

Rioux, M. (1950). Remarques sur la notion de culture en anthropologie. Revue d'histoire de l'Amérique française, 4(3), 311-321.

https://doi.org/10.7202/801651ar d'utilisation que vous pouvez consulter en ligne.

https://apropos.erudit.org/fr/usagers/politique-dutilisation/ 


\section{REMARQUES SUR LA NOTION DE CULTURE EN ANTHROPOLOGIE}

Dans son "Histoire de l'Education dans l'Antiquite", Henri Marrou écrit: "Le mot culture: il faut lui conserver le sens spécifiquement français de forme personnelle de la vie de l'esprit en évitant de le prendre comme font abusivement nos ethnographes au sens de l'allemand Kultur qui signifie civilisation"'. Mon but est d'examiner, dans cet article, ces deux notions de culture et de civilisation; c'est avec le désir de voir s'uniformiser la terminologie de l'histoire et de l'anthropologie culturelle, ces deux points de vue différents pris sur la même réalité, que ces quelques remarques sont formulées.

De nos jours, à cause des emprunts constants que se font la langue courante et la langue technique, il devient de plus en plus difficile de savoir si tel terme est pris dans son sens spécialisé ou s'il appartient a la langue des honnêtes gens. D'autre part, chaque discipline se rétrécissant toujours en se spécialisant davantage, elle en vient à employer des termes qui ne sont pas c smpris des usagers ordinaires de la langue française ni même des spécialistes qui œuvrent dans des domaines connexes. Comme les termes culture et civilisation appartiennent au vocabulaire de toutes les sciences de l'homme, de la sociologie, de l'histoire, de la philosophie et à celui de tous les honnêtes gens, il devient extrêmement difficile d'en arriver à une entente sur le sens précis qu'il convient de donner à ces termes. Mais il semble que si une discipline particulière a fait d'une notion son objet spécifique d'étude, qu'il convient, à tout le moins dans des études. techniques, de suivre l'usage de cette discipline. Or, l'anthropologie culturelle, qui existe comme discipline, sinon comme appellation, depuis au moins un siècle a fait de la culture son objet propre de recherche. Il conviendrait donc d'examiner ce que cette discipline

1. Henri Marrou, Histoire de l'éducation dans l'Antiquité, (Paris, 1948), 465. 
entend quand elle parle de culture. Mais auparavant, quelques remarques s'imposent.

En posant en principe que les disciplines qui se veulent scientifiques doivent tenter d'éviter toute définition ou tout concept coloré par un quelconque élément de valeur, il ne semble pas faire de doute que tous ceux qui s'occupent de l'homme d'un point de vue objectif s'accorderont là-dessus. Or il est clair que la plupart des acceptions de culture et de civilisation sont teintées d'affectivité et d'ethnocentrisme. Ne faudrait-il pas alors les épurer pour en faire disparaître tout élément qui risquerait d'infléchir les jugements que ceux qui s'occupent des sciences de l'homme portent sur les phénomènes qu'jls étudient? D'autre part quand une définition colle aux faits - comme nous allons essayer de le montrer pour la notion de culture, dans son sens anthropologique moderne - est-il bien juste de vouloir la rejeter parce qu'elle n'existe pas dans la langue de tel chercheur? D'ailleurs, pour ce qui a trait au mot culture, il n'y aurait aucune honte, pour nous, de langue française, à emprunter ce mot aux Allemands, car nous ne ferions alors que reprendre notre bien. Tonnelat n'affirme-t-il pas que le "Kultur" allemand est un calque direct du français "culture"2 ? Et, d'autre part, le terme culture, tel qu'il est employé aujourd' ıui par les anthropologistes anglo-saxons, est loin d'avoir le même sens que le "Kultur" allemand; il est vrai que les Allemands ont beaucoup influencé la formation de la théorie anthropologique contemporaine - Boas, Lowie, Sapir, Goldenweiser étaient tous sinon Allemands du moins de langue allemande mais on ne saurait nier qu'il y a entre les deux conceptions des différences importantes. On pourra peut-être objecter encore que le mot culture fait double emploi avec le mot civilisation et qu'il ne fait d'ailleurs pas partie du bagage conceptuel de l'Ecole Sociologique française. Nous allons tenter de prouver qu'il ne fait pas double emploi avec le mot de civilisation et que l'idée, sinon le mot, se retrouve chez Durkheim et ses disciples. Comme Lalande ${ }^{8}$ lui-même emprunte ses définitions de culture et de civilisation à Mauss et à

2. E. Tonnelat, Kultur in "Civilisation, le mot et l'Idee (Centre International de Synthèse, Paris, 1930), 61.

3. Lalande, Vocabulaire technique et critique de la philosophie: aux mots culture et civilisation. 
monsieur Marrou, ce sont les définitions de ces deux auteurs que nous allons confronter avec celles de l'anthropologie culturelle; Mauss nous servira d'indicateur pour le mot civilisation et Marrou pour le mot culture.

Dans l'usage courant de l'anthropologie culturelle le mot civilisation n'a pas beaucoup cours. Il est quelquefois employé d'une façon assez lâche comme synonyme d'une des acceptions de "culture" et ne comporte alors aucun élément de valeur; il a alors le même sens que Marrou lui donne: "cette notion de civilisation déterminée, relative, notion purement descriptive, vidée de tout élément de valeur"4; cet emploi est toutefois assez rare et n'est pas des meilleurs auteurs. Quelques anthropologistes l'emploient dans son sens étymologique (civis, habitant d'une cite) pour désigner les sociétés urbaines. D'autres, par opposition à société primitive, archaïque ou "folk society" utilisent quelquefois, société civilisée; cet emploi marque une idée d'évolution et quelquefois aussi, d'ascension et de progrès. Une acception que l'on aimerait voir plus répandue chez les anthropologistes de langue anglaise et même chez ceux de langue française, c'est celle de Mauss; elle ne fait pas double emploi ni avec le mot de culture ni aucun autre terme employé en anthropologie. Mauss définit ainsi le "fait de civilisation" - qui est lui-même une espèce de fait social: "Les phénomènes de civilisation sont ainsi essentiellement internationaux, extranationaux. On peut donc les définir en opposition aux phénomènes sociaux, spécifiques de telle société: ceux des phénomènes sociaux qui sont communs à plusieurs sociétés plus ou moins rapprochées"s. Il définit ensuite une civilisation: "C'est un ensemble suffisamment grand de phénomènes de civilisation, suffisamment nombreux, eux-mêmes suffisamment importants tant par leur masse que par leur qualite; c'est aussi un ensemble, assez vaste par le nombre de sociétés qui les présentent; autrement dit: un ensemble suffisamment grand et suffisamment caractéristique pour qu'il puisse sigaifier, évoquer à l'esprit une famille de sociétés"'. Cette

4. Henri Marrou, "Culture, Civilisation, Décadence", Revue de Synthèse, XV: 139.

5. Marcel Mauss, "Les Civilisations, Eléments et Formes" in Civilisation, le Mot et l'Idée (Centre International de Synthèse, Paris, 1930), 86.

6. Ibid., 88-89. 
notion s'articule très bien avec la théorie anthropologique contemporaine; elle n'est pas normative et désigne justement une catégorie de faits pour lesquels on chercherait en vain une désignation chez les anthropologistes modernes. La définition de Mauss est partitive; elle ne s'applique qu'à définir un fait de civilisation, une civilisation et non pas la civilisation comme phénomène général; ainsi défini, le fait de civilisation serait une espèce particulière de fait de culture, c'est-à-dire un fait culturel qui voyage, qui déborde les frontières géographiques de ce qu'on est convenu d'appeler une culture. Sans vouloir préjuger de la question qu'on peut se poser pour savoir si fait culturel et fait social ont la même extension, on peut toutefois affirmer que dans le contexte de la théorie anthropologique, le mot de culture a plus d'extension que celui de civilisation, au sens où Mauss l'entend. Une culture, envisagée comme un tout, comprend et les faits qui sont particuliers à cette société et ceux qui sont communs à plusieurs sociétés; les deux espèces de phénomènes sont essentiellement culturels; les derniers peuvent être désignés comme des faits de civilisation.

Si nous passons au mot culture nous constatons que l'idée de culture a toujours évoqué l'idée d'effort en vue d'obtenir que le quelque chose qui fait l'objet de l'attention de celui qui cultive croisse et donne des fruits. Appliquée à l'homme, cette notion a toujours voulu signifier le processus du développement des potentialités humaines. Si nous comparons l'homme aux autres animaux nous nous rendons compte qu'un des traits les plus distinctifs de cet être, c'est son manque de spécialisation biologique qui nécessite une période d'éducation qui, comparée à celle des autres animaux, est extrêmement prolongée. L'homme, en naissant, est tout à fait dépourvu de tout; il faut qu'il apprenne tout ce qui fera de lui un homme et non seulement un être humain, mais un citoyen de tel ou tel Etat, de telle région, de telle ville, de telle classe sociale, de telle famille. Toutes ces idées qu'il acquiert par l'enseignement, par l'imitation, par l'éducation formelle et diffuse et par osmose, si l'on peut dire, c'est ce que l'anthropologiste appelle culture; c'est une classe très générale de phénomènes qui s'oppose à d'autres classes aussi générales; c'est tout ce qui n'est pas organique, tout ce qui n'est pas physique, tout ce qui n'est pas géographique et qui fait que tel homme est ce qu'il est, se range dans le domaine de la culture. Envisagé du point 
de vue de la société elle-même, le concept de culture s'applique encore à cet héritage cumulatif que de génération en génération les hommes se transmettent, qu'ils enrichissent, transforment, appauvrissent et quelquefois dilapident. L'aventure humaine, comme le disait Pascal, peut être représentée comme un seul homme qui apprendrait sans cesse. Cet héritage social que chaque société se transmet d'une génération à l'autre en y mettant elle-même sa marque, c'est le produit de ce processus dont nous venons de parler. Culture peut être dit du processus et du produit. Envisagée sous le premier aspect la culture humaine nous apparait donc comme une nature acquise, une nature seconde qui se surajoute à la nature innée de l'homme et qui lui permet de développer ses potentialités et de vivre pleinement sa vie d'homme. La culture humaine consiste donc en formes acquises de comportements, de sentiments et d'idées. Il serait trop long de nous demander ici si la culture consiste uniquement en schèmes mentaux qui informeraient les comportements et les réalisations matérielles (position idéaliste) ou si elle ne se définit pas plutôt comme étant une qualité du comportement humain, détectable dans les objets matériels, les coutumes et les idéaux (position réaliste).

Ce que nous voulons retenir pour les fins du présent article, c'est que le concept de culture, entendu dans sa plus grande extension, peut se définir comme étant le processus d'éducation, de mise en valeur et d'intégration de l'homme tout entier. Si nous envisageons le résultat général de ce processus général, on peut dire que le concept de culture s'applique aux formes acquises de technique, de comportement, de sentiment et d'idées que les indi vidus ont acquis comme membres de la société humaine ainsi qu'aux institutions sociales dont ils se servent pour atteindre leur bien commun. De plus, une culture s'entendrait d'un système donné de comportements et d'idées, d'idéaux et de conduites ainsi que des produits de ces activités qui sont communes à ou professées par un groupe d'hommes donné. Cette conception anthropologique de la culture humaine diffère-t-elle de la conception "personnaliste" de monsieur Marrou? Nous ne le croyons pas. Evidemment la conception anthropologique implique un certain déterminisme mais c'est un déterminisme limité et qui s'efforce de tenir compte des réalités sociales sans nier la part que l'individu prend dans l'élaboration d'un système culturel donné. 
L'individu peut surmonter et surmonte ce déterminisme culturel puisque toute culture, même la olus primitive, étant dynamique, il faut donc reconnaitre que les individus surmontent l'inertie culturelle du milieu et transforment l'héritage qu'ils ont reçu des générations précédentes. Si l'anthropologie met l'accent sur ce qu'il y a de conmun entre les hommes d'une société donnée, c'est d'abord parce que c'est son objet propre et que, d'une façon absolue, on ne peut nier cet ordre de phénomènes. Que chaque individu, considéré comme produit fini, soit différent de tous et chacun des autres individus, l'anthropologie ne le nie pas; mais ce n'est pas tant la différence ultime, les caractères individuants qui l'intéressent que le genre prochain qui intervient dans l'équation de chaque individu qui l'intéresse; ce n'est pas tant non plus l'individu atypique, anormal, en tant que tel, qui l'intéresse, que l'individu normal; ce n'est pas tant une réalisation extraordinaire d'un individu que les cuvres, les comportements, les idées, les sentiments des individus ordinaires d'une société donaée. Il ne semble pas y a voir de conflit entre la théorie anthropologique et le personnalisme, ni même l'existentialisme. L'individu reste théoriquement libre de se créer, de se faire c smme il le veut. Mais qui niera que l'on ne peut s'échapper tout à fait de son milieu social? Celui qui s'en échappe totalement c'est le psychopathe dont Blondel et Wallon, entre autres, ont décrit les comportements et qui, ayant perdu toutes ses attaches avec la société et la culture du groupe humain dont il fait partie, ne peut être considéré que comme malade mental; ce n'est que dans ce cas que l'on peut observer de l'individuel à l'état pur. Celui qui ordinairement est considéré comme grand homme, dans quelque domaine que ce soit, est justement l'individu qui, ayant absorbé tout ce que la société pouvait lui offrir dans un domaine donné, va de l'avant, énonce d'autres hypothèses, d'autres idées, fabrique d'autres engins, essaie de nouvelles combinaisons. Si ces inventions et ces nou veautés sont agréées du reste de la société, elles deviennent à leur tour partie de la culture de cette société, sont enseignées, transmises, modifiées et suivent le même processus que les autres traits culturels ont connu.

Monsieur Marrou, dans son excellent essai, s'arrête longuement sur la notion de culture préparatoire. Selon lui, cette culture préparatoire s'entend du "contenu de l'éducation, entendue au sens large, et non uniquement scolaire: on fait entrer dans cette culture prépa- 
ratoire non seulement ce qu'enseigne l'école mais encore les lectures, les méditations, les expériences de tout ordre que l'enfant et surtout l'adolescent, le jeune homme ajoute aux leçons de ses maîtres"'. Cette notion est très voisine de celle de l'anthropologie qui reconnaît comme étant primordiales les premières expériences de l'adolescence et même de l'enfance; on se rend de plus en plus compte que même les premiers mois de la vie des individus sont très importants du point de vue de la socialisation de l'être humain. Pour expliquer les différents types de personnalité qui, il est vrai, varient aussi à l'intérieur d'une culture donnée, mais qui varient encore plus de culture à culture, on en est venu à mettre l'accent sur cette "culture preparatoire" et à se demander, par exemple, si étant données telles disciplines de l'enfance, tel type de personnalité n'aura pas tendance à s'ensuivre. La structure de la personnalité de l'individu est fortement influencée par la façon dont il apprend à vivre sa vie d'homme et ce, dès les premiers mois de son existence. Ruth Benedict, par exem ple, a étudié l'influence de la puériculture sur la genèse du caractère national des peuples européens. Voici ce qu'elle dit d'une pratique russe: "L'emmaillottement tel qu'il se pratique en GrandeRussie apprend à l'enfant à faire de bonne heure une distinction nette entre la spontanéité intérieure, qui ne semble pas souffrir des entraves extérieures, et les restrictions du milieu, que l'on estime être le prix à payer pour conserver la spontanéité intérieure"s. Ces influences que l'individu subit et qui ne font pas partie de l'éducation formelle, ni de l'instruction, aident à rendre compte de la formation de l'individu et quand elles sont générales, du caractère d'une nation. Monsieur Marrou, comme le fait l'anthropologiste, lui aussi passe du processus au produit, de la culture entendue comme moyen d'humaniser l'homme-animal, au résultat de ce processus. "La culture désigne souvent non pas l'état, la qualité d'homme cultivé, mais le contenu de l'intelligence d'un homme cultivé, ses connaissances, ses capacités, et aussi, quand on ne le considère pas seulement au repos mais encore en action, ses diverses formes d'activité".

7. Ibid., 137.

8. Ruth Benedict, L'Etude des traditions culturelles dans le monde civilisé (UNESCO, Paris, 1948), 8.

9. Ibid., 138. 
Le sens de monsieur Marrou est restrictif; quand il parle de la forme personnelle de la vie de l'esprit il semble qu'il n'ait en vue que certains individus à l'intérieur d'une société donnée, ceux qui ont acquis le plus de notions littéraires historiques et scientifiques. L'auteur s'explique là-dessus quand il en vient à discuter les acceptions françaises et allemandes des termes culture et civilisation; il termine ses remarques en déclarant que l'opposition qui doit exister entre ces deux termes doit être située non pas dans le rapport spirituel-matériel, mais bien dans celui: vie individuelle-milieu collectif. Nous suivons l'auteur quand il nous dit ce que l'opposition entre les deux termes ne doit pas être mais non pas quand il propose ce que cette opposition devrait être. D'ailleurs, s'agit-il bien d'opposition? Ne serait-ce pas plutôt des notions complémentaires? Nous proposons plutôt de conserver la définition que Mauss donne de civilisation ainsi que la définition de culture de l'anthropologie culturelle car ces concepts, particulièrement le dernier, sont tellement riches et expliquent tellement bien la réalité qu'il n'y a aucune raison de les écarter. Quant à la notion de vie personnelle, ne seraitil plus juste de faire appel à la psychologie et à la psychanalyse, de lui emprunter le concept de personnalité et de l'intégrer dans les autres sciences de l'homme?

Ainsi, les sciences sociales, en empruntant, d'une part, certaines des idées de l'anthropologie physique qui explique l'homme biologique et en s'assimilant, d'autre part, certaines notions de la psychologie qui traite de l'individu, en tant que tel, se forment une vue extrêmement riche de l'homme - vue qui débouche aux deux pôles où leur objet s'arrête. Cette notion de personnalité peut être très utile non seulement a la psychologie pour laquelle elle est un concept-clé mais aussi pour l'anthropologie et pour l'histoire, pour toutes les disciplines qui étudient l'homme sous quelque aspect que ce soit. Si toutes ces études doivent aboutir à une explication du comportement de l'homme réel, en chair et en os, et non plus seulement étudier les rónlisations humaines en quelque sorte "réifiées" et vidées de leur dynamisme, le concept de personnalité apparait comme étant une des clés essentielles du problème. Le "Dictionary of Sociology" de Fairchild donne de personnalité la définition suivante: "That dynamic organization of ideas, attitudes, and habits which is built upon the foundation of the biologically inherited 
psycho-physical mechanisms of a single organism and of socially transmitted culture patterns, and which embodies all the adjustments of this individual's motives, desires and purposes to the requirements and potentialities of his social and sub-social environments". Linton donne une définition plus concise; pour lui, c'est la somme organisée des processus et des états psychologiques d'un individu ${ }^{10}$. Quelle que soit la définition que l'on adopte, cette notion de personnalité est extrêmement riche et répond à la question de monsieur Marrou qui veut connaitre "la forme personnelle de la vie de l'esprit."

Monsieur Marrou nous fait, d'autre part, remarquer que le mot culture reproduit l'histoire du mot civilisation ou l'on en vient à passer du processus au résultat et de parler de civilisation en excluant tout élément de valeur; peut-être faudrait-il continuer l'analogie et vider aussi le mot culture, du moins dans les sciences de l'homme, de son contenu normatif. Monsieur Marrou entend, par culture "la forme particulière que l'idéal de la vie intellectuelle revêt pour les hommes d'une société donnée". Si Monsieur Marrou entendait par intellectuelle tout le domaine des idées sans distinction quant à leur noblesse ou leur objet - l'idée de couteau à manche voisinant avec l'idée de la quatrième dimension - il se rapprocherait de ceux des anthropologistes qui définissent la culture exclusivement en termes d'idées. Mais l'auteur entend par vie intellectuelle, vie "supérieure de l'esprit" et sa notion de culture se rétrécit d'autant. Si nous comprenons bien la pensée de l'auteur, il recherche ce que l'on considère à un moment donné, dans une société donnée, l'idéal de la vie intellectuelle. Le texte de monsieur Marrou nous autorise à croire que l'idéal de la vie intellectuelle signifie ce que la société se représente comme la forme la plus parfaite de la vie de l'esprit. Il est évident que cette notion elle-même fait partie de ce que nous avons inclus dans la catégorie culture; ce n'est qu'un des éléments des "modèles idéaux" d'une société. Ces modèles varient donc de société à société. Chez les Iroquois paiens de Brantford, il semble que ce soit la connaissance des mythes, de l'histoire, des rites, de nombreux dialectes qui constitue la forme supérieure de la vie de l'esprit; dans une petite île canadienne-française, il semble qu'en termes pure-

10. Ralph Linton, The Cultural Background of Personality, 84 . 
ment locaux, l'idéal de la vie intellectuelle soit la connaissance de l'histoire de l'Ile, des généalogies et d'anecdotes. Dans une société plus complexe, il faudra distinguer entre l'idéal commun à toute la société et l'idéal de différents groupes; l'idéal des professeurs au Collège de France, des anciens de Polytechnique et des boutiquiers des quais de la Seine n'est certainement pas le même et varie aussi dans le temps. Mais cette notion n'a rien de personnel; c'est un des modèles culturels d'une société ou d'une partie d'une société.

Si l'on veut étudier, comme monsieur Marrou veut le faire, une classe sociale ou un groupe d'individus, les notions de culture et de civilisation, dans leur sens anthropologique, suffisent pour étudier l'intellectuel-type d'une époque donnée. A ce sujet, monsieur Marrou donne des conseils extrêmement pertinents. Ainsi au lieu de se contenter des modèles idéaux (ideal patterns), c'est-à-dire de ce que les individus d'une société donnée sont censés faire, il étudiera aussi ce que ces individus font en réalité; ce qui veut dire qu'en plus de chercher ce que les élèves d'un certain district sont censés apprendre il faudra encore se rendre compte de ce qu'ils apprennent en réalité. Il y a toujours un décalage assez prononcé entre ce qu'on fait et ce qu'on est censé faire, entre les modèles de pensée et les modèles de conduite qui guident les individus. La méthode que l'auteur a suivie pour explorer la vie de saint Augustin, les anthropologistes la suivent pour explorer la vie de quelques-uns des individus qu'ils choisissent dans une société pour connaître cette société et pour se rendre compte des effets d'une culture donnée sur tels individus qui y participent; ils ne s'attendent pas à retrouver chez cet individu toute la culture de la société à laquelle il appartient ni encore à n'y trouver que du culturel ou du social; il y a un plan proprement individuel que l'anthropologiste ne nie pas mais qu'il laisse à d'autres le soin d'étudier et d'expliquer.

Les études que monsieur Marrou a lui-même entreprises prouvent jusqu'à quel point culture et personnalité s'entrepénètrent. Inutile, d'une part, d'essayer de connaître la culture d'un groupe d'hommes, d'une société, d'une nation sans s'adresser aux individus, sans connaitre comment cette culture influence les individus et comment les individus transforment cette culture; ce n'est qu'ainsi que l'on pourra obtenir une image vraiment significative et réelle d'une société. D'autre part, l'historien ou le psychologue ne pourra 
jamais avoir une représentation adéquate de l'individu qu'il étudie s'il ne tient pas compte du milieu culturel considéré comme un tout et dans lequel son sujet baigne depuis sa naissance. Société, culture et personnalité sont des réalités, non pas opposées, mais complémentaires et qui s'expliquent l'une par l'autre. C'est pourquoi les disciplines qui étudient un aspect ou l'autre de ces trois catégories devraient, autant que faire se peut, se tenir au courant de ce qui se fait dans les secteurs voisins pour donner à leurs méthodes et à leurs vues toute la richesse que cette intégration apporte.

Marcel Rioux, Attache au Service d'anthropologie, Musée National du Canada. 